Article

\title{
Early Inoculation of Microbial Suspension in Suckling Piglets Affects the Transmission of Maternal Microbiota and the Associated Antibiotic Resistance Genes
}

\author{
Caroline S. Achard ${ }^{1,2}$, Veronique Dupouy ${ }^{3}{ }^{\circledR}$, Laurent Cauquil ${ }^{1}\left(0\right.$, Nathalie Arpaillange $^{3}$, \\ Alain Bousquet-Melou ${ }^{3}{ }^{-}$, Nathalie Le Floc' $h^{4, \dagger}$ and Olivier Zemb ${ }^{1, *,+}$ \\ 1 Génétique Physiologie et Systèmes d’Elevage (GenPhySE), Université de Toulouse, Institut National De \\ Recherche Pour L'agriculture, L'alimentation Et L'environnement (INRAE), Institut National Polytechnique \\ de Toulouse (INPT), École nationale vétérinaire de Toulouse (ENVT), F-31320 Castanet Tolosan, France; \\ cachard@lallemand.com (C.S.A.); laurent.cauquil@inrae.fr (L.C.) \\ 2 Lallemand SAS, 19 rue des Briquetiers, BP 59, 31702 Blagnac CEDEX, France \\ 3 Innovations thérapeutiques et résistances (INTHERES), Université de Toulouse, INRAE, ENVT, \\ F-31300 Toulouse, France; v.dupouy@envt.fr (V.D.); n.arpaillange@envt.fr (N.A.); \\ a.bousquet-melou@envt.fr (A.B.-M.) \\ 4 Physiologie, Environnement et Génétique pour l'Animal et les Systèmes d'Élevage (PEGASE), Institut \\ national de recherche pour l'agriculture, l'alimentation et l'environnement (INRAE), Institut Agro, \\ 35590 Saint-Gilles, France; nathalie.lefloch@inrae.fr \\ * Correspondence: Olivier.zemb@inrae.fr \\ + These authors contributed equally to the work.
}

Received: 14 August 2020; Accepted: 7 October 2020; Published: 13 October 2020

\begin{abstract}
Antibiotic resistance of microbes thriving in the animal gut is a growing concern for public health as it may serve as a hidden reservoir for antibiotic resistance genes (ARGs). We compared 16 control piglets to 24 piglets fed for 3 weeks with S1 or S2 fecal suspensions from two sows that were not exposed to antibiotics for at least 6 months: the first suspension decreased the erythromycin resistance gene $\mathrm{erm} B$ and the aminoglycoside phosphotransferase gene conferring resistance to kanamycine (aphA3), while the second decreased the tetracycline resistance gene tet $L$, with an unexpected increase in ARGs. Using 16S RNA sequencing, we identified microbial species that are likely to carry ARGs, such as the lincosamide nucleotidyltransferase $\ln u B$, the cephalosporinase $\operatorname{cep} A$, and the tetracycline resistance genes tet $G$ and tet $M$, as well as microbes that never co-exist with the tetracycline resistance gene tet $Q$, the erythromycin resistance gene erm $G$ and aphA3. Since $73 \%$ of the microbes detected in the sows were not detected in the piglets at weaning, a neutral model was applied to estimate whether a microbial species is more important than chance would predict. This model confirmed that force-feeding modifies the dynamics of gut colonization. In conclusion, early inoculation of gut microbes is an interesting possibility to stimulate gut microbiota towards a desirable state in pig production, but more work is needed to be able to predict which communities should be used.
\end{abstract}

Keywords: competitive exclusion; microbiota; piglet; antibiotic resistance genes

\section{Introduction}

The prevalence of antibiotic resistance genes (ARGs) in livestock is country-specific. For example, Chinese pigs have more ARGs encoding resistance to chloramphenicol, gentamycin B, kanamycin and neomycin than their French and Danish conspecifics [1]. Country-specific ARG profiles also exist within Europe, where microbiota of pigs from Italy have more of the streptomycin-resistant 
mutation $\operatorname{str} A$ than those from France, Denmark or Sweden [2]. Furthermore, the country-specific differences of ARGs in livestock seem to penetrate the human gut. Indeed the ARGs in Spanish, Danish and U.S. citizens mirror the antibiotics used in livestock in each of these countries, such as streptomycin-resistance which is more prevalent in Spain than in Denmark [3]. More indirect evidence comes from the nisin-producing operons in bacterial isolates from human, porcine and bovine hosts, revealing extensive bacterial transmission between humans and pigs [4]. ARGs in livestock may therefore become a public health issue if they are transferred to pathogenic bacteria that inhabit the human gut.

In response to the increase in antibiotic resistance, the European Union banned antibiotics as growth promoters for livestock in 2006, and data suggest that the impact of this ban on the performances of growing pigs is relatively minor at the country-level in Denmark [5], or in carefully controlled groups [6,7]. However, a long-term study conducted at the University of Kentucky showed that stopping antibiotics could also be detrimental to reproductive performance since sows not treated with antibiotics farrowed and weaned fewer pigs per litter [8]. Therefore, excluding antibiotics from the nursery is far from trivial.

As a matter of fact, the 2006 ban on fattening animals might not be sufficient to decrease antibiotic resistance, as seen by Spanish Methicillin-resistant Staphylococcus aureus strains that became more resistant to multiple antibiotics between 2009 and 2018 [9]. Indeed, ARGs may incur almost no fitness cost for the bacteria. For example, Campylobacter spontaneously evolved a resistance against macrolides and was not outcompeted by its sensitive kin in an antibiotic-free environment [10]. This lack of fitness cost implies that ARGs might persist in the commensal microbiota years after the antibiotic treatment [11,12]. In this context, long-lasting resistance genes found in the maternal environment are an issue because young animals are likely to inherit antibiotic-resistant microbes from their mothers and the environment. Indeed, the microbiota of rabbit kits that have been adopted just after birth is closer to the microbiota of the lactating doe than to the microbiota of the biological mother [13], and piglets are sensitive to their surrounding microbes as well [14]. Therefore, long-lasting resistance genes are a logical target to fight against antibiotic resistance inherited by piglets from lactating sows.

The idea of using a complex microbial community to remediate against a specific bacterial species was first described in 1973 [15] to outcompete pathogenic Salmonella in broilers. Competitive exclusion was also successfully adapted to the exclusion of antibiotic-resistant E. coli in broilers [16,17] and in mice after an antibiotic treatment [18]. Nowadays, human fecal transplantation using complex microbial communities can cure Clostridium difficile infections [19], and lyophilization protocols have been developed to facilitate its routine use [20]. However competitive exclusion studies using complex microbial communities remain relatively scarce for mammalian livestock. In pigs, Salmonella could be outcompeted using complex cultures grown in vitro [21,22], but using competitive exclusion has been reported to sometimes increase the antibiotic resistance of E. coli [23] or to have no effect in vivo [24].

In this paper, we investigate the potential of complex microbial communities to stimulate the microbiota of piglets from ARG-carrying sows and, especially, to outcompete bacteria carrying ARGs. The complex microbial communities were orally delivered. We measured antibiotic resistance and transposase gene abundance, Enterobacteriacae resistance to tetracycline or sulphonamide, and the taxonomic composition of the fecal microbiota of the piglets before weaning.

\section{Materials and Methods}

The experiment was conducted at the France's new National Research Institute for Agriculture, Food and Environment (INRAE) Pig Physiology and Phenotyping Experimental Facility (UE3P) in Saint-Gilles (France) in compliance with the ethical standards of the European community (Directive 2010/63 EU). Six Large-White $x$ Landrace sows were selected from the INRAE herd and were inseminated with Piétrain semen. All the sows involved in the experiment and their litter were housed in the same building and were gestating and lactating at the same time. The pigs were housed in farming conditions as authorized by the French regulation on animal experimentation for experimentations conducted 
with an agronomic purpose. At 106 days of gestation, sows were moved to the farrowing room with natural (windows) and artificial light. They were kept in individual farrowing crates $(2 \times 2.5 \mathrm{~m})$ equipped with a trough and a drinker. The floor of the farrowing pens was made of slatted plastic. The farrowing crates were equipped with 2 infrared heat bulbs for piglets. The ambient temperature was kept at $24{ }^{\circ} \mathrm{C}$ to $25^{\circ} \mathrm{C}$ in the lactation rooms with heating and ventilation. Farrowing was induced by an intramuscular injection of prostaglandin F $2 \alpha$ ( $2 \mathrm{~mL}$ of Dinolytic, Zoetis, France) on day 114 of gestation. Usual farm practices for newly farrowed piglets were individual identification by tagging, iron injection, tail docking, and castration of male piglets. Cross-fostering, if needed, was performed intra-treatment within 2 days after birth but piglets selected for blood and feces samplings were maintained with their biological mother.

\subsection{Donor Sows and Preparation of the Microbial Suspensions}

Two donor sows (first parity for Sow 206 generating the S2 suspension and third parity for Sow 268 generating the $\mathrm{S} 1$ suspension) were selected based on their low abundance of sulfonamideand tetracycline-resistant Enterobacteriacae. On the morning of the days of oral inoculation of the piglets, fresh feces were collected from the sows and $20 \mathrm{~g}$ were immediately mixed with $110 \mathrm{~mL}$ of anoxic physiological salt solution at $0.9 \% \mathrm{NaCl}$ in a screw-cap $125-\mathrm{mL}$ bottle equipped with a septum. After vigorous shaking, the suspension was made anoxic by three cycles of vacuum/filling with $\mathrm{N}_{2}$. The last cycle ended with an overpressure of 500 mbar. The suspension was then immediately administered to the piglets by force-feeding.

\subsection{Lactating Sows and Experimental Piglets}

Four gestating sows (three were primiparous and one was multiparous) were selected on the basis of their relatively high abundance of sulfonamide- and tetracycline-resistant Enterobacteriacae $(31 \% \pm$ $18 \%$ and $36 \% \pm 15 \%$ ). After farrowing, the piglets remained with their biological mother during the experiment. Force-feeding took place every two days from Day 2 to Day 23 after birth by placing $2 \mathrm{~mL}$ of the above-described microbial suspension near the soft palate using a plastic syringe. Within each litter, five piglets were force-fed with $2 \mathrm{~mL}$ of saline (Control), three piglets received the suspension from donor sow S1, and three piglets received the suspension from donor sow S2. Piglets were weaned at 4 weeks. No antibiotic treatment was administrated either to the sows or the piglets.

\subsection{Feces Sampling and DNA Preparation}

Fresh feces were sampled from the lactating sows and from their piglets by rectal stimulation at Day 14 and at four weeks just before being separated from their mother. The donor sows were sampled 1 and 2 months before the experiment and once at the end. The samples were immediately stored at $-80^{\circ} \mathrm{C}$. The DNA was extracted from $50 \mathrm{mg}$ of porcine stool using a bead-beating approach, as previously described [25], and stored at $-80^{\circ} \mathrm{C}$.

\subsection{Analysis of the Antibiotic Resistance Genes via qPCR}

The DNA concentration was measured using the Quant-iT TM PicoGreen TM dsDNA Assay Kit, according to the manufacturer's protocol (Invitrogen) and subsequently diluted to $10 \mathrm{ng} / \mu \mathrm{l}$.

High throughput real-time qPCR was performed using the Biomark microfluidic system from Fluidigm (San Francisco, CA, USA) using a 96.96 Dynamic Array ${ }^{\mathrm{TM}}$ Integrated Fluidic Circuit (IFC). Pre-amplification of the samples, chip loading and qPCR reactions in nanoliter volumes were performed according to the manufacturer's protocol. A pre-amplification step was applied to all samples for all primer sets except the 16S rRNA primer set. Briefly, $13 \mathrm{ng}$ of total DNA were submitted to 14 PCR cycles using the PreAmp Master Mix (Fluidigm, San Francisco, CA, USA) and a mix of primers (50 nM final concentration). Pre-amplified samples were diluted five-fold after an exonuclease treatment. The diluted pre-amplified samples and the primer sets were loaded in a 96.96 IFC using an IFC Controller HX (Fluidigm, San Francisco, CA, USA). The Biomark thermal protocol was as follows: 
a thermal mix step $\left(50{ }^{\circ} \mathrm{C}, 2 \mathrm{~min} ; 70{ }^{\circ} \mathrm{C}, 30 \mathrm{~min} ; 25^{\circ} \mathrm{C}, 10 \mathrm{~min}\right)$; a hot start $\left(50{ }^{\circ} \mathrm{C}, 2 \mathrm{~min} ; 95^{\circ} \mathrm{C}, 10 \mathrm{~min}\right)$; 35 cycles of PCR $\left(95^{\circ} \mathrm{C}, 15 \mathrm{~s} ; 60^{\circ} \mathrm{C}, 60 \mathrm{~s}\right)$; a final melting phase $\left(60^{\circ} \mathrm{C}\right.$ to $\left.95^{\circ} \mathrm{C}\right)$.

The list of primers can be found in Table S5. The quantity of each gene (in arbitrary units) was extrapolated using a generated standard curve with Fluidigm real-time PCR analysis software (v4.3.1).

\subsection{Analysis of the Culturable Enterobacteriacae}

Feces were sampled from the donor sows 2 months before the experiment, at Day 14 and at Day 27. Feces from the lactating sows and from their piglets were sampled at Day 14 and Day 27 (weaning). Feces $(5 \mathrm{~g})$ from each animal were blended, homogenized in $45 \mathrm{~mL}$ of peptone water including $30 \%$ glycerol, and stored at $-80{ }^{\circ} \mathrm{C}$ until use. Ten-fold serial dilutions of the homogenate were prepared, and 100- $\mu$ l samples of the dilutions were spread on MacConkey agar (Sigma, M8302) in order to obtain ten isolates of Enterobacteriacae. The antibiotic susceptibility of Enterobacteriacae isolates was tested by plating a $24 \mathrm{~h}$ pure culture onto Mueller-Hilton agar alone or supplemented with $16 \mu \mathrm{g} / \mathrm{mL}$ tetracycline or $128 \mu \mathrm{g} / \mathrm{mL}$ sulfamethoxazole, according to the E. coli epidemiological cut-off values defined by Eucast [26]. Every test included a resistant and sensitive control strain to certify that the antibiotic was active. The proportion of sulfonamide- or tetracycline-resistant Enterobacteriacae was calculated for each animal.

\subsection{Analysis of the Microbiota Composition Using the $16 S$ rRNA Gene}

The V3-V4 region was amplified from diluted genomic DNA with the primers F343 (CTTTCCC TACACGACGCTCTTCCGATCTTACGGRAGGCAGCAG) and R784 (GGAGTTCAGACGTGTGCTCT TCCGATCTTACCAGGGTATCTAATCCT) using 30 amplification cycles with an annealing temperature of $65{ }^{\circ} \mathrm{C}$ (an amplicon of $510 \mathrm{bp}$, although the exact length varies depending on the organisms). This region was chosen because it has proved useful for several studies on the variability of the microbiota in pigs exposed to different surrounding microbes and diets [25,27]. Because the V3 kit of Illumina enables paired 300-bp reads, the ends of each read are overlapped and can be stitched together to generate extremely high-quality, full-length reads of the entire V3 and V4 region in a single run. Each pair-end sequence was assembled using Flash software v1.2.6 [28] with at least a 10-bp overlap between the forward and reverse sequences, allowing 10\% mismatch. Single multiplexing was performed using an in-house 6 bp index, which was added to R784 during a second PCR with 12 cycles using forward primer (AATGATACGGCGACCACCGAGATCTACACTCTTTCCCTACACGAC) and reverse primer (CAAGCAGAAGACGGCATACGAGAT-index-GTGACTGGAGTTCAGACGTGT). The resulting PCR products were purified and loaded onto the Illumina MiSeq cartridge according to the manufacturer's instructions. The quality of the run was checked internally using PhiX, and each pair-end sequence was then assigned to its sample with the help of the previously integrated index using bcl2fastq provided by Illumina. The sequences were submitted to the Short-Read Archive [29] with accession number SRP124929.

The sequences were filtered for quality (length $>150 \mathrm{bp}$, homopolymers $<10,100 \%$ unambiguous bases) and reassigned to each sample using barcodes, which were also trimmed. The resulting sequences were clustered into operational taxonomic units (OTU)s using USEARCH [30] and counted in each sample to create a table of relative abundance of each OTU across the samples, yielding between 12,726 and 18,198 high-quality sequences per sample. The representative sequence of each OTU was classified on the Ribosomal Database Project trainset16, reformatted to be compatible with USEARCHv11 (https://www.drive5.com/usearch/manual/sintax_downloads.html), and the taxonomic affiliations with a confidence level lower than 0.8 were labeled 'unclassified'. The counts were rarefied at 12,726 sequences using the rarefy_even_depth function from the phyloseq package [31].

\subsection{Statistical Analysis}

Statistical analyses were performed using Rstudio software with R (v3.3). The difference between the ratios of resistant Enterobacteriacae was modeled by a binomial logistic regression in order to take 
the small number of strains per sample $(<11)$ into account. The differences in ARGs between donor and lactating sows were evaluated for the genes detected in every sample by a Wilcoxon rank sum test corrected for false discovery rate using the Benjamini-Hochberg procedure. The dissimilarities between the bacterial communities of the sows and their piglets were evaluated by the Bray-Curtis distance after rarefaction by the minimal number of reads per sample (i.e., 12,720). The normalized OTU abundance table was used to discriminate the piglets of the three groups (S1, S2 and control) using principal component analysis and discriminant analysis (DAPC) [32]. Briefly, in DAPC, the samples are described in orthogonal axes using a principal component analysis, and a discriminant analysis is applied on the first components. The significance of the separation between samples is quantified with the built-in a-score function with 1000 simulations. Briefly, the a-score compares the separation achieved to the separations than can be achieved when the groups are randomly attributed. The minimal number of OTUs to discriminate the groups in order to obtain significant separation between S1 and S2 was determined by the stepwise addition of the OTUs that contributed the most to the DAPC until significance, using the a-score function with 1000 permutations, is reached. The neutral model was independently fitted to piglets with the same inoculation and the same mother (i.e., 12 sets) using the script provided by Burns et al. [33], and the resulting goodness-to-fit was analyzed by ANOVA. The impact on the ARGs was evaluated with a Friedman test based on the medians.

The linear and non-linear relationships between the selected OTUs and ARGs were characterized on a filtered OTU table so that the total count of each OTU across the samples is higher than 1000 (i.e., $0.07 \%$ of the total counts), which was merged with the ARG dataset using the sample Id. We then computed the maximal information coefficient index using the application provided with Java SE 1.7.0_02 [34] with the percentage of the records necessary to have data in them for both variables before those variables are compared (cv) and the exponent $(\alpha)$ parameters set to 0.6. To account for the baseline correlation bound to occur between any two vectors, the empirical null distribution of the maximal information coefficient was computed using 1000 random permutations of the relative abundances within the variables. The maximal value of the null distribution was set as the significant threshold for the real dataset, i.e., the dataset before randomization. The relationships were labeled as linear when the regression coefficient was higher than 0.5 . They were labeled as non-linear when the regression coefficient was negative, and the nonlinearity coefficient was higher than 0.3 . The dataset at 14 days and the dataset at weaning were analyzed separately to avoid relationships due to the impact of age.

\section{Results}

\subsection{The Donor and Lactating Sows Had Different Resistance Profiles}

The prerequisite when attempting a targeted competitive exclusion is that the inoculum is depleted in the target. We checked this prerequisite by measuring the proportion of resistant Enterobacteriacae over the total count of Enterobacteriacae by plating the feces of the sows on selective medium: the proportion of resistant Enterobacteriacae was indeed lower in the donor sows that generated suspensions S1 and S2 (sow 268 and sow 206) than in the four lactating sows (sow 512, sow 709, sow 710 and sow 722 ) (6 $\pm 8 \%$ vs. $36 \pm 15 \%$, Table S1). We also measured the ARGs by quantitative PCR in six samples from the donor sows and the 12 samples from the lactating sows: unfortunately, ARGs in the donor and the lactating sows were not significantly different when correcting for the false discovery rate (Table S2), even though some heterogeneity was observed prior the experiment (Figure S1).

\subsection{Oral Inoculation of Microbial Suspension Affected the ARGs in Piglet Feces}

Two sows that were not exposed to antibiotics for at least 6 months generated the S1 and S2 suspensions that were fed from Day 2 to Day 23 to the piglets born from the four lactating sows: force-feeding the S1 suspension impacted 15 ARGs in the piglets, while force-feeding the S2 suspension impacted eight ARGs in the piglets compared to the control piglets (Tables 1 and 2, respectively). 
Some ARGs were impacted at both sampling dates, while others were impacted at one date only. For example, the $\mathrm{S} 1$ suspension decreased the abundance of the aminoglycoside nucleotidyltransferase gene aadE_2 at 14 days, but this effect was no longer observed by the date of weaning (i.e., 4 weeks). It should be noted that $a a d E \_2$ tended to be lower in donor sows than in lactating sows according to a Wilcoxon test ( $p=0.052$, Table S2). Remarkably, other ARGs such as the glycopeptide resistance gene vanTG or $\ln u B$ were increased by the S1 suspension in both sampling dates (Table 1 ).

Table 1. Impact of the suspension from donor sow 268 (S1) on the abundance of antibiotic resistance genes (ARGs) and related genes in the piglets at 14 days and at 4 weeks (median values). The ARG abundances are normalized by the abundance of the $16 \mathrm{~S}$ rRNA gene. The numbers are not between 0 and 1 because the ARGs were pre-amplified, whereas the 16S rRNA genes were not (see Materials and Methods section, Table S3 and Figure S5). The ARGs aphA3 and ermB are underlined because they are decreased by the inoculation of the microbial suspension $\mathrm{S} 1$.

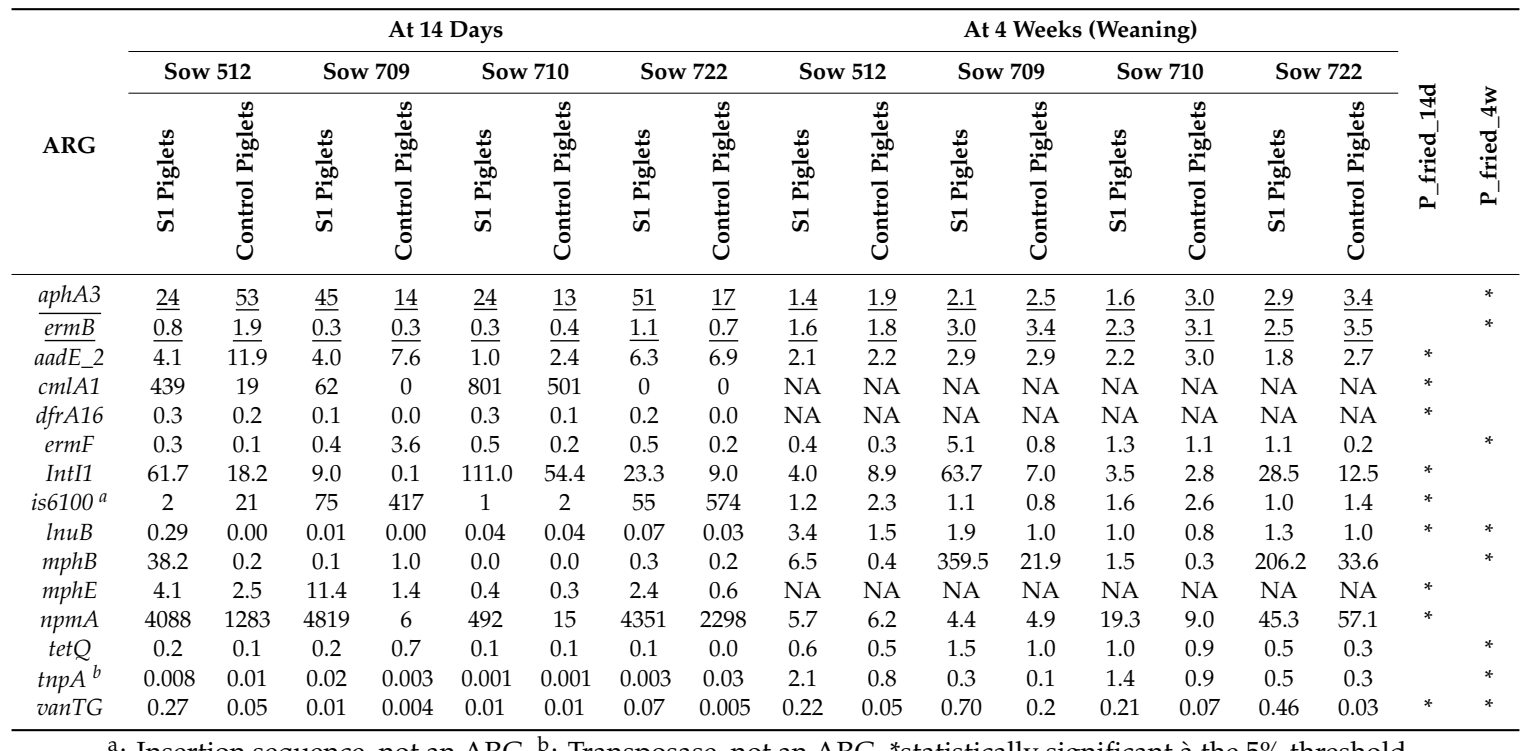

Table 2. Impact of the suspension from donor sow 206 (S2) on the abundance of ARGs and related genes in the piglets at 14 days and at 4 weeks (median values). The ARG abundances are normalized by the abundance of the 16S rRNA gene. The numbers are not between 0 and 1 because the ARGs were pre-amplified, whereas the 16S rRNA genes were not (see Materials and Methods section, Table S4 and Figure S6). The ARG tet $L$ is underlined because it is decreased by the inoculation of the microbial suspension $\mathrm{S} 2$ of the piglets.

\begin{tabular}{|c|c|c|c|c|c|c|c|c|c|c|c|c|c|c|c|c|c|c|}
\hline \multirow[b]{3}{*}{$\begin{array}{l}\text { Primer } \\
\text { Set ID }\end{array}$} & \multicolumn{8}{|c|}{ At 14 Days } & \multicolumn{8}{|c|}{ At 4 Weeks (Weaning) } & \multirow{3}{*}{ 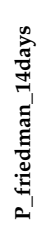 } & \multirow{3}{*}{ 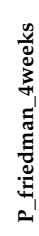 } \\
\hline & \multicolumn{2}{|c|}{ Sow 512} & \multicolumn{2}{|c|}{ Sow 709} & \multicolumn{2}{|c|}{ Sow 710} & \multicolumn{2}{|c|}{ Sow 722} & \multicolumn{2}{|c|}{ Sow 512} & \multicolumn{2}{|c|}{ Sow 709} & \multicolumn{2}{|c|}{ Sow 710} & \multicolumn{2}{|c|}{ Sow 722} & & \\
\hline & 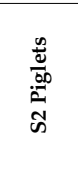 & 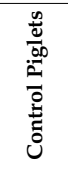 & $\begin{array}{l}\frac{n}{0} \\
\frac{0}{00} \\
\tilde{D} \\
\text { N }\end{array}$ & 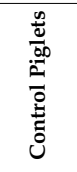 & 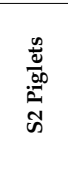 & 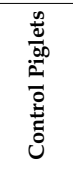 & $\begin{array}{l}\frac{0}{0} \\
\frac{0}{00} \\
\overrightarrow{0} \\
\tilde{\omega}\end{array}$ & $\begin{array}{l}\frac{0}{0} \\
\frac{0}{00} \\
0 \\
0 \\
0 \\
0 \\
0 \\
0\end{array}$ & 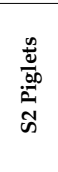 & 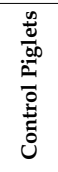 & 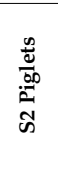 & 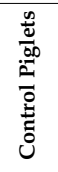 & 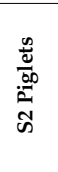 & $\begin{array}{l}\frac{n}{0} \\
\frac{0}{00} \\
0 \\
0 \\
0 \\
0 \\
0 \\
0\end{array}$ & $\begin{array}{l}\frac{D}{0} \\
\frac{0}{00} \\
\overrightarrow{0} \\
\tilde{\omega}\end{array}$ & 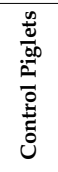 & & \\
\hline $\operatorname{aad} A$ & 311.85 & 69.3 & 54.75 & 51.18 & 465.4 & 83.50 & 12.08 & 4.26 & 0.28 & 0.19 & 0.15 & 0.16 & 0.40 & 0.08 & 0.62 & 0.22 & * & \\
\hline$a p h 2 I b$ & 5217.6 & 7.2 & 1.3 & 1.1 & 69.9 & 10.8 & 201.7 & 17.7 & 167 & 179 & 218 & 33.2 & 14.9 & 3.5 & 147 & 129 & * & \\
\hline$d$ frA $A 16$ & 0.56 & 0.19 & 0.07 & 0.04 & 0.14 & 0.12 & 0.23 & 0.03 & NA & NA & NA & NA & NA & NA & NA & NA & * & \\
\hline is $6100^{a}$ & 65.36 & 21.3 & 1257.7 & 416.8 & 2.78 & 2.37 & 643.2 & 574.4 & 3.56 & 2.31 & 2.15 & 0.82 & 1.43 & 2.58 & 1.25 & 1.36 & * & \\
\hline $\operatorname{lnu} C$ & 2.83 & 1.74 & 0.50 & 1.68 & 1.06 & 1.00 & 1.80 & 1.12 & 1.87 & 1.03 & 1.62 & 1.07 & 1.47 & 0.86 & 1.12 & 1.00 & & * \\
\hline tetW.1 & 0.45 & 0.27 & 0.24 & 0.21 & 0.17 & 0.11 & 0.25 & 0.20 & 2.08 & 1.03 & 2.39 & 1.47 & 1.25 & 0.93 & 1.78 & 1.28 & * & * \\
\hline
\end{tabular}


Interestingly, the decreased abundance of a specific ARG in the suspension was not always a guarantee of a decrease in that ARG in the force-fed piglets. The difficulty of predicting the success of the competitive exclusion is striking for the 16S rRNA methyltransferase npmA against aminoglycosides, which was present in each lactating sow, undetectable in the S1 and S2 suspensions but not modified by force-feeding the suspensions ( $p=0.32$ according to the Wilcoxon test) (Tables S2 and S3). The complexity of predicting the success of the ARG exclusion from the ARGs in the donor and the lactating sow is presented in Figure S2.

Perhaps unsurprisingly, force-feeding the microbial suspensions S1 and S2 did not impact the same genes, except for the insertion sequence is6100. For example, the $\mathrm{S} 1$ suspension successfully reduced the abundance of aph $A 3$ and ermB at weaning, whereas the S2 suspension did not, even though it harbored similar levels of these genes (Table S2). Both suspensions also increased two to five different ARGs at weaning (ermF, $\operatorname{lnu} B, \operatorname{mpm} B, \operatorname{tet} Q, \operatorname{tnp} A$ and vanTG vs. $\ln u C$ and tet $W)$. While undesired, this increase in ARGs in the force-fed piglets supports the hypothesis that inoculating an anaerobic microbial suspension resulted in foreign microbial species colonizing the gut of the piglets. In total, the abundance of eight and two genes in the feces of piglets at weaning were modified by the inoculation of the microbial suspensions S1 and S2, respectively $(a p h A 3, \operatorname{ermB}, \operatorname{ermF}, \operatorname{lnu} B, m p m B$, tet $Q, \operatorname{tnp} A$ and vanTG in Table $1 ; \ln u C$ and tet $W$ in Table 2$)$.

\subsection{Oral Inoculation of Microbial Suspension Temporarily Affected the Antibiotic Resistance of Enterobacteriacae}

Force-feeding the microbial suspension temporarily changed the abundance of the Enterobacteriacae, which were isolated from the feces of piglets and subsequently tested for their sulfonamide and tetracycline resistance. Surprisingly, the proportion of resistant Enterobacteriacae was higher in the force-fed piglets at 2 weeks of age, even though the donor sows had less resistant Enterobacteriacae than the lactating sows at the beginning of the experiment. Unfortunately, the proportion of tetracycline-resistant Enterobacteriacae in the donor sows increased from $6 \pm 8 \%$ to $50 \pm 28 \%$ during the experiment (Tables S1 and S2), and ended up being comparable to the lactating sows at the end of the experiment $(48 \pm 37 \%)$ even though no antibiotics were used. The impact of force-feeding on resistant Enterobacteriacae disappeared by the time of weaning (Figure S3), at which point only the impact of the lactating sow remained significant (Figure S4).

\subsection{Oral Inoculation of Microbial Suspension Affected the Vertical Transmission of Bacteria}

Inoculating the S1 or S2 suspensions affected the fecal microbiota of the piglets collected at weaning (4 weeks). Indeed, the discriminant analysis on the principal components that combined the samples from Days 14 and 27 revealed a significant impact of both inocula compared to the control piglets ( $p=0.036$ and 0.047 , based on 1000 random permutations; Figure 1).

To determine if the inoculation resulted in OTUs that are more frequent in piglets than expected by random colonization, the frequency of each OTU was compared to its mean abundance in piglets exposed to the same microbial metacommunity, i.e., applying the neutral model to the piglets sharing the same treatment (S1, S2 or saline) and the same litter. Interestingly, the neutral model describes the species distribution relatively well for control piglets under each sow $\left(R^{2}=0.28 \pm 0.09\right)$, but never for S1 or S2 piglets $\left(R^{2}=0.08 \pm 0.15\right.$ and $0.04 \pm 0.13$ ) (Figure 2 and Figure S7), confirming that a different dynamic is taking place when piglets are orally inoculated. The poor fit of the neutral model implies that the community assembly rules are different when feeding the microbial suspensions S1 or S2 instead of feeding saline water. The goodness-to-fit of the model was not dependent on the age of the piglets ( $p=0.21$, according to ANOVA). It should also be noted that the model was adjusted from an extrapolated 'piglet metacommunity' because the microbes naturally colonizing the gut of the piglets are different from the communities in the sows ( $p=0.001$, according to ADONIS), hindering the direct use of the neutral model. In fact, $73 \%$ of the OTUs that were always present in the control piglets were undetectable in their lactating sows (Figure S8). Perhaps unsurprisingly, the OTUs relevant to the 
discrimination of the inoculated piglets are often undetectable or rare in the donor sows (Figure S9). For example, OTU12 is the OTU that contributes the most to the separation between the control, S1 and S2 piglets on the second axis (Figure 1), and is overrepresented in S1 piglets ( $p=0.004$, according to a Wilcoxon test) but merely represented less than $0.02 \%$ in the donor sows (Figure S9).

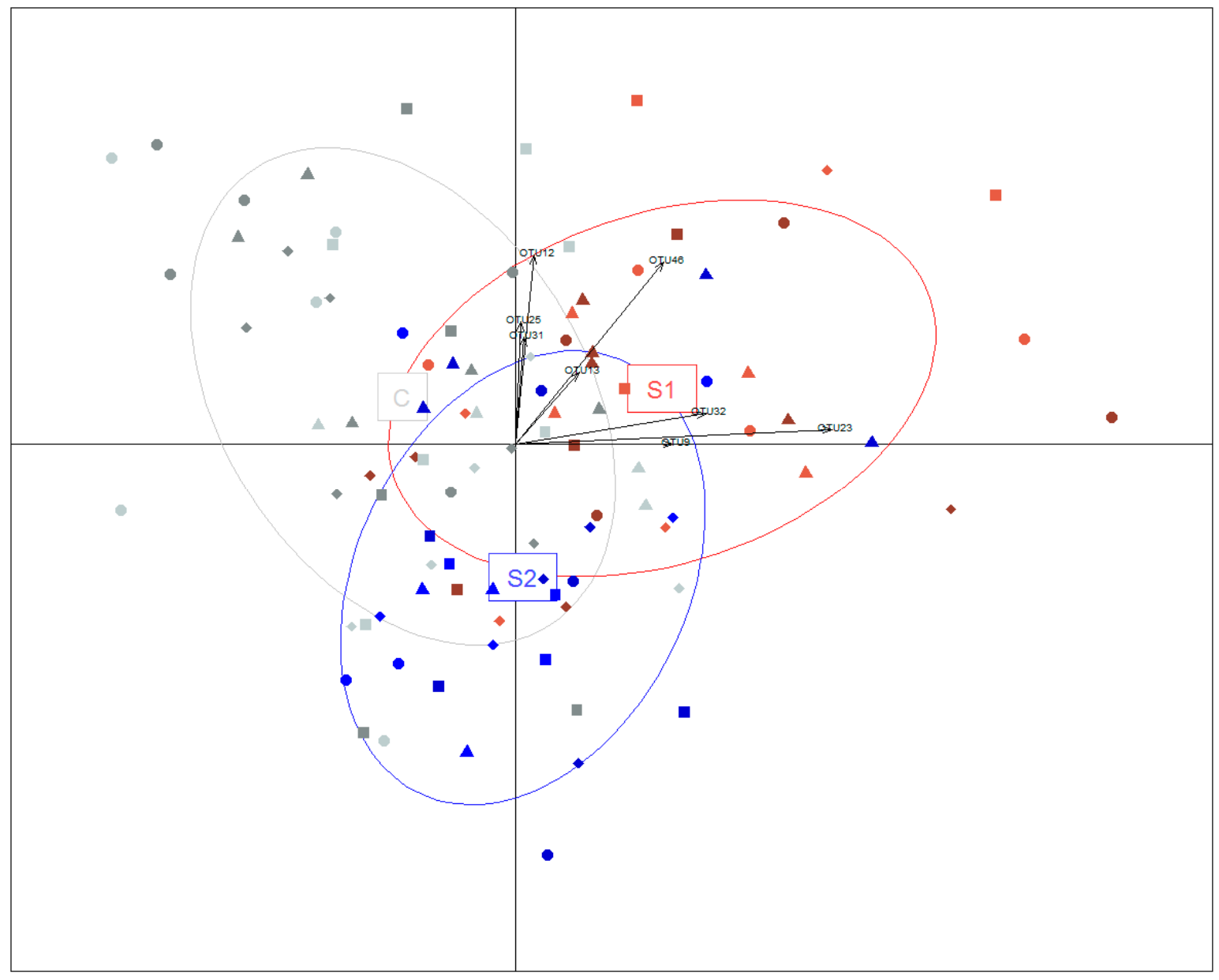

Figure 1. Multidimensional representation of the microbiota of piglets inoculated with either suspension S1, suspension S2 or the control piglets inoculated with saline water. The inset represents the variability taken into account by the principal component analysis (91\%). The control piglets are in gray while the piglets force-fed with the S1 or S2 microbial suspensions prepared from the donor sows with low ARG levels are in red and blue. The contributions of the eight operational taxonomic units (OTUs) of interest are indicated by arrows. Piglets from sows 512, 709, 710 and 722 are represented as squares, circles, triangles and diamonds, respectively. The samples at 14 days are shown in lighter shades and the samples at weaning are shown in darker shades. 


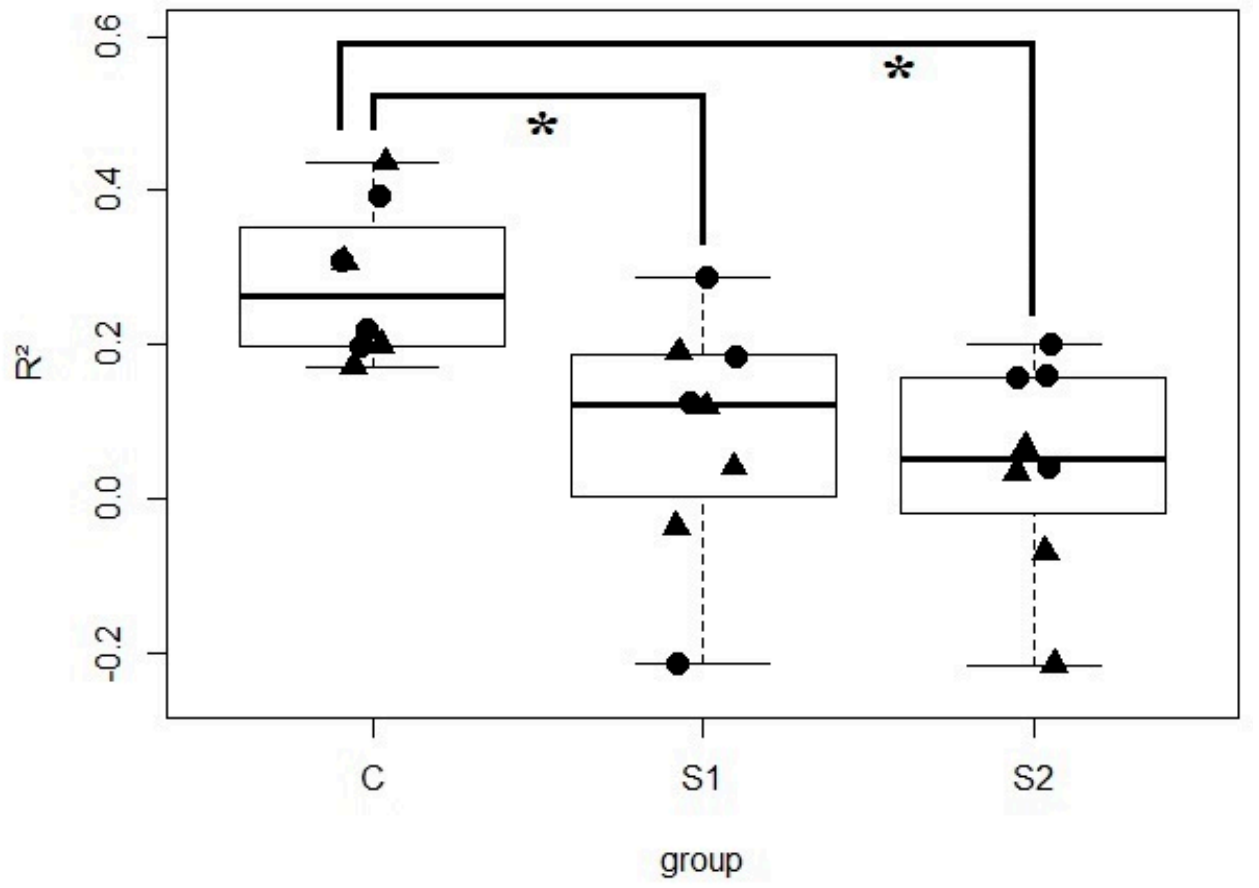

Figure 2. $\mathrm{R}^{2}$ values of the neutral model fitting the $\mathrm{S} 1, \mathrm{~S} 2$ and control piglets. Circles are the 14-day-old piglets and triangles are the piglets at 4 weeks (weaning). Star $\left(^{*}\right)$ indicate that the $\mathrm{R}^{2}$ values are significantly different (at the 5\% threshold of the ANOVA) between the control piglets and the piglets force-fed with the S1 or S2 microbial suspensions prepared from the donor sows with low ARG levels, illustrating that the inoculation modified the assembly rules of the microbes.

\subsection{Relationships between OTUs and ARGs}

In order to pinpoint the OTUs carrying or excluding ARGs, the maximal information coefficient index was used to evaluate the linear and co-exclusion relationships between OTUs and ARGs. To avoid capturing relationships due to the impact of age, data at both dates were analyzed separately. At 14 days, the only three significant relationships involving ARGs were OTU23 (Bacteroides)-catA1 (non-coexistence), OTU49 (Roseburia)-cmlA1 and OTU6 (Bacteroides)-cepA. Many more relationships were detected at weaning (Table 3 ), at which point the maximal information coefficient was significant for 852 pairs among the 31,626 pairs analyzed, which included 532 linear correlations, 149 co-exclusion relationships and 171 other significant relationships (Figures S10-S12). We observed the OTU6 (Bacteroides)-cepA relationship again, along with other single linear OTU-ARG correlations pointing towards probable ARG carriers: $\ln u B$, tet $G$, tet $M$ and erm $G$ were linked to OTU43 (Turicibacter), OTU198 (unclassified Bacteroidia), OTU35 (Lactobacillus) and OTU5 (unclassified Ruminococcaceae), respectively. The tet 32 gene was linearly linked both to OTU7 and OTU4458, which share $99 \%$ identity and $100 \%$ query coverage with the uncultured clone $\mathrm{p}-4570-4 \mathrm{Wb3}$ found in pigs [35]. Interestingly, we also identified OTUs that never co-exist with specific ARGs, suggesting a possible exclusion mechanism of the microbe carrying the ARGs by the OTU. For example, we observed OTUs connected to numerous ARGs by co-exclusion, such as OTU229 (unclassified Bacteroidales) and OTU868 (Clostridium_sensu_stricto), which never co-exist with the aadE-aphA3-ermG-ermB cluster (Figure S9). The antibiotic resistance genes tend to cluster together, with up to eight different ARGs in the cluster containing erm $G$ and aphA3 (Figure S9), but the clusters are not always associated with OTUs. For example, mefA and tetQ form a cluster of ARGs with no linear relationship to any of the OTUs, in contrast with the cluster of tet40, erm $G, I n u C$, aadE, tet $W, a p h A 3$ and ermB that includes several OTUs. As expected, the measurements using different primers for the same gene (such as aphA3 and aphA3) cluster together. The OTUs also form clusters containing two to 35 OTUs (data not shown). 
Table 3. Remarkable links between OTUs and ARGs at weaning.

\begin{tabular}{|c|c|c|c|c|}
\hline $\begin{array}{c}\text { Type of } \\
\text { Relationship }\end{array}$ & ARGs or IS & Targeted Antibiotics & $\begin{array}{c}\text { Linked } \\
\text { OTU }\end{array}$ & $\begin{array}{c}\text { Taxonomy of the Linked } \\
\text { OTU }\end{array}$ \\
\hline \multirow{18}{*}{$\begin{array}{l}\text { Linear relationship: } \\
\text { proportionality } \\
\text { between OTU and } \\
\text { ARG abundances }\end{array}$} & \multirow{2}{*}{$\operatorname{aph} A 3$} & \multirow{2}{*}{ Kanamycin } & 131 & unclassified Clostridia \\
\hline & & & 2302 & unclassified Clostridiales \\
\hline & серA & Cephalosporin & 6 & Bacteroides fragilis \\
\hline & tet $G$ & Tetracycline & 198 & unclassified Bacteroidia \\
\hline & \multirow{2}{*}{ пртA } & \multirow{2}{*}{ Aminoglycoside } & 10 & unclassied Clostridiales \\
\hline & & & 12,054 & unclassied Clostridia \\
\hline & tetM & Tetracycline & 35 & Lactobacillus \\
\hline & $\ln u B$ & Lincosamide & 43 & Turicibacter \\
\hline & \multirow{2}{*}{$a n t 6-I b$} & \multirow{2}{*}{ Streptomycin } & 28 & unclassified Fusobacteriaceae \\
\hline & & & 120 & unclassified Clostridiales \\
\hline & $\begin{array}{l}\text { dfrA1-sul2-intl1- } \\
\text { aadA-aadA2-strB }\end{array}$ & \multicolumn{3}{|c|}{ Diaminopyrimidine-Sulfonamide-Aminoglycoside } \\
\hline & blaTEM- tetR-tet $A$ & $\begin{array}{c}\beta \text {-lactam } \\
\text { antibiotics-Tetracylcline }\end{array}$ & & \\
\hline & $m e f A-t e t Q$ & Macrolide-Tetracycline & & \\
\hline & $\begin{array}{l}\text { tetO-tet40-aadE-aphA3- } \\
\text { tetW-ermG-ermB-lnuC }\end{array}$ & $\begin{array}{l}\text { Tetracycline-Streptomycin- } \\
\text { Kanamycin-Macrolide- } \\
\text { Lincosamide-Streptogramin B }\end{array}$ & & \\
\hline & \multirow{2}{*}{ tet32 } & \multirow{2}{*}{ tetracycline } & 7 & unclassified Clostridia \\
\hline & & & 4458 & unclassified Firmicutes \\
\hline & Intl1 & - & 51 & unclassified Clostridiales \\
\hline & ermB & $\begin{array}{l}\text { Macrolide-Lincosamide- } \\
\text { Streptogramin B }\end{array}$ & 5 & unclassified Ruminococcaceae \\
\hline \multirow{7}{*}{$\begin{array}{l}\text { Co-exclusion } \\
\text { relationship: the } \\
\text { gene was never } \\
\text { observed together } \\
\text { with the OTU }\end{array}$} & \multirow{4}{*}{ ermB and erm $G$} & \multirow{4}{*}{$\begin{array}{l}\text { Macrolide-Lincosamide- } \\
\text { Streptogramin B }\end{array}$} & 229 & Unclassified Bacteroidales \\
\hline & & & 100 & Unclassified Bacteroidales \\
\hline & & & 193 & Prevotella \\
\hline & & & 868 & Clostridium_sensu_stricto \\
\hline & \multirow{2}{*}{ tet $L$} & \multirow{2}{*}{ Tetracycline } & 57 & unclassified Firmicutes \\
\hline & & & 12 & unclassified bacterium \\
\hline & ermF & $\begin{array}{l}\text { Macrolide-Lincosamide- } \\
\text { Streptogramin B }\end{array}$ & 5858 & unclassified Clostridia \\
\hline
\end{tabular}

\section{Discussion}

This study revisits the concept of competitive exclusion first explored by Nurmi [15]. We demonstrate that the abundance of specific bacterial species is modified by the repeated inoculation of a microbial suspension, which in turn impacts the abundance of antibiotic resistance genes. The success of the ARG exclusion undoubtedly depends on the composition of the inoculated community, since inoculating the S1 and S2 suspensions had markedly different results for ARG and $16 \mathrm{~S}$ rRNA genes in piglets.

\subsection{Promoting Sow-to-Piglet Transmission}

In our experiment, the piglets were in continuous microbial exposure with their lactating mother through the environment and the nursing. Despite this contact, force-feeding $2 \mathrm{~mL}$ of an anaerobic microbial suspension three times a week from Day 2 to Day 23 was enough to modify the gut microbial communities so that the species distribution would no longer fit the neutral model. Thus, the microbial imprint acquired through birth or early investigation of the environment can be overridden by a relatively modest but early inoculation of microbes, even when piglets are not raised in isolators. 
Several studies excluded the maternal contact from their offspring. For example, piglets in isolators were "humanized" by injecting $3 \mathrm{~mL}$ of human fecal suspension [36]. Moreover, piglets in a non-sterile room fed with an automatic feeding system and inoculated with complex microbial communities clustered together [37], suggesting that the inherited microbiota may be replaced by force-feeding the young animals with a fecal suspension. However, these studies ignored the maternal contact even though radioactivity fed to lactating sows can be retrieved in their feces and their piglets, leading to an estimated ingestion of $8.5 \mathrm{~g}$ to 20.9 of feces and bedding per day [38]. The contribution of this ingestion of feces to the colonization of the gut is corroborated by the fact that the succession and stabilization events reported in naturally-reared outdoor pigs does not occur when the regular immigration of microbes is shut down by rearing the piglets in isolators, with no contact with the feces of their mother [39]. More recently, inoculation of piglets that still live under their mother showed that changing the microbiota did not require the elimination of all other microbial contacts to be effective: feeding the cross-bred piglets with a fecal suspension derived from another breed resulted in a microbial community enriched in five microbial species [40]. The importance of early-life microbial contact is also illustrated by the ease with which microbes can be inoculated in newly-hatched chicks $[17,41]$. Taken together, these results show that a complex microbe community free of pathogenic bacteria could be implanted by appropriate exposure of the young animals to complex anaerobic microbial communities. A similar observation was made in rabbits [42]. We confirm here that this tool can also be used in piglets living under their mothers to change the rules that underlie microbial assembly in piglets, as shown by the failure of the neutral model to capture the species distribution in force-fed piglets.

The age at which the piglets are susceptible to their surrounding microbial communities is still unknown, but the pre-weaning period seems to be the most well-adapted: (a) co-housed piglets suddenly develop very similar communities at around 2-3 weeks of age [14]; (b) feeding yeast to piglets before weaning impacts the average daily weight gain of the piglets, whereas feeding yeast after weaning does not [43]; (c) feeding a complex community to piglets between 10 and 18 days of age changed the microbial community in a way that increased robustness when the piglets are submitted to an early weaning at 21 days [40]; and (d) cross-fostering an obese typical Chinese piglet breed and a lean Western breed demonstrated the impact of the nursing mother on the piglets' microbiota and interleukin 10 [44]. In other species, the microbial environment in early life is also more important than the microbial imprint at birth, as revealed by the cross-adoption of 1-day-old rabbits [13]. Humans may appear as the exception to the rule since babies delivered by C-section still had less vaginal bacteria after 1 month [45], but the vaginal signature represents only a small fraction of the microbial community. For the record, pigs are still mildly susceptible to their microbial environment after weaning, as illustrated by the impact of poor hygiene on microbiota of 8-week-old pigs [27]. Late susceptibility is also observed in humans where competitive exclusion against Clostridium difficile was successful in human adults when ingesting encapsulated microbiota [20]. At any rate, exclusion against maternal ARGs in piglets has to be performed after birth and before weaning to limit the diffusion of ARGs on the farm. Taken together, these results suggest that force-feeding piglets could counter the drift of the microbiota exposed to antibiotics after birth [46]. The transmission of antibiotic-resistant microbes to piglets that we observed might also partly explain why conventional and organic farms had similar ARG levels [2]. Indeed, some ARG-carrying microbes (such as Megasphaera elsdenii) might arise in piglets after weaning, even if their pen is in a separate room from their mother and no antibiotics are used [47], so that organic farms may carry over ARGs when they introduce piglets raised under ARG-carrying sows.

\subsection{The Impact of Anaerobic Microbes on Antibiotic Resistance Genes}

Initially, none of the ARGs were significantly depressed in the two donor sows compared to the four lactating sows, possibly because of the heterogeneity observed in the four lactating sows, which also translated in piglets with contrasted ARG abundance backgrounds. For example, the control piglets of Sow 512 had up to 80-fold less $m p h B$ than the control piglets from Sow 722 (Table S3). 
Nevertheless, the piglets fed with the selected communities had more resistant Enterobacteriacae at Day 14 than the controls, as well as different communities at weaning, showing that the inoculation had an impact, albeit not always a desirable one. Interestingly, the exclusion of the probable ARG carrier could be caused by microbes that were phylogenetically distant. For example, our results showed that OTU5 (unclassified Ruminococcaceae) is probably the carrier of ermB, which is favored by tylosin when used as a growth promoter in pig production [48].

The largest ARG cluster is the tetO-tet40-aadE-aphA3-tet W-ermG-ermB-lnuC cluster. The ermB gene was previously described with aadE and aphA3 in Enterococcus faecium from poultry manure and sewage [49]. Interestingly, ermB is "non-coexistent" with microbes such as OTU229 (unclassified Bacteroidales) and OTU868 (Clostridium_sensu_stricto). OTU229 shares 99\% identity with the clone T1WK15A found in Canadian piglets whose ermB abundance did not increase upon exposure to antibiotics [50], in contrast to previous observations [48]. A larger study involving 681 Danish pigs at slaughter showed a correlation between $\operatorname{ermB}$ and their lifetime exposure to tetracycline, but the model was only able to explain $42 \%$ of the variance [51], suggesting that other factors play a role in the ermB response upon antibiotic exposure. It can only be speculated as to whether or not one of these factors is the composition of the maternal microbiota, which could explain the high background resistance that is sometimes observed in non-medicated pigs [52].

Exclusion led by microbes from a taxonomic order different from the ARG carrier has practical implications. Indeed, OTUs excluding ermB do not belong to the Ruminococcaceae family that includes OTU5, the probable carrier of ermB. Similarly, an experiment in mice showed that Barnsiella (bacteroidales) prevents the colonization from vancomycin-resistant enterococcus (lactobacillales) [18]. The practical implication is that replacing an ARG-resistant microbe by its sensitive kin may not be the optimal strategy. In fact, the inoculation of sensitive Megasphaera elsdenii to exclude the resistant strain was not visible at Day 45 despite six inoculations between Day 3 and Day 34 [47].

It should be noted that maintaining anaerobic conditions probably increases the chances of a successful inoculation. For example, anaerobic microbes from the complex community are more efficient at excluding the vancomycin-resistant enterococcus than their aerobic kin in mice. Previous successful experiments of fecal transplants in piglets also maintained anaerobic conditions [40]. In our opinion, maintaining anaerobic conditions like those we described in the Materials and Methods section may be the key to success since anaerobes become dominant in piglets after $24 \mathrm{~h}$, reaching $3 \times$ $10^{9} \mathrm{CFU} / \mathrm{g}$ [53].

Surprisingly, the large ARG clusters were similar to the clusters observed in China, even though Chinese and French pigs have country-specific ARG profiles [1]. For example, the dfrA1-sul2-intl1aadA-aadA2-strB cluster was also reported in China, as was the association between mefA and tet $Q$ and between aphA3 and tetW [54]. Conversely, our results showed striking differences with Chinese data that cannot be explained by technical aspects. Indeed, sul2 is not directly connected to any OTU in our dataset. Even when lowering our threshold to 1\%, sul2 becomes connected to OTU51 (unclassified Clostridiales), whereas it is linked to a member of the Xanthomonadales in China. Such differences in the likely bacterial host for sul2 might arise from the mobility of this genetic element, which has been found in very diverse bacteria, ranging from the Pseudomonadales order (Acinetobacter) to the Enterobacteriales order (Proteus mirabilis) [54]. Mobile genetic elements may in fact underpin several associations observed in the clusters. For example, the ermB-aadE and ermB-aphA3 associations are observed on Transposon5385 and Transposon1545 [55] that may be transferred between bacteria, and were reported in 33 different genera [56]. In this context, inoculating complex communities rather than single strains could be preferable because it may simultaneously exclude all the species carrying the transposon and, consequently, hinder the transmission of mobile genetic elements carrying genes such as ermB.

The smaller ARG-OTU clusters validate the method used in this study. For example, the OTU6 identified as a probable carrier for cepA shares $100 \%$ identity with the Bacteroides fragilis strain NCTC 9343, which carries a chromosomal beta-lactamase cepA [57]. Similarly, OTU35 associated with tetM in 
our study is identical to Lactobacillus salivarius JCM1046 whose genome harbors a single copy of an integrated conjugative transposon with the tetracycline resistance gene tet $M$ [58].

\section{Conclusions}

In conclusion, feeding complex microbial communities may be advantageous for excluding clusters of antibiotic-resistant genes in young piglets, but great care should be given to avoid an accidental increase in ARGs, as highlighted in this study. This is challenging because the final concentration of ARGs could not be predicted from the simple absence of the target in the donor sow. Further work is therefore needed to improve our understanding and our predictions of competitive exclusion.

Supplementary Materials: The following are available online at http:/www.mdpi.com/2076-2607/8/10/1576/s1, Table S1: Counts of tetracycline and sulfamide-resistant Enterobacteria. Table S2: ARG abundances in the six samples from the donor sows and in the 12 samples from the lactating sows. Table S3: Significance of the exclusion and relative abundance of targeted genes in the S1 and control piglets at weaning. Table S4: Significance of the exclusion and relative abundance of targeted genes in the S2 donor. Table S5: List of primers used in this paper. Figure S1: Control of ARG variability in the donor and lactating sows 3 months before the experiment. Figure S2: Relative abundance of ARGs in S1 and S2 piglets at weaning (as the ratio of the ARG in the control piglets) vs. the abundance of ARGs in the donor sow (as the ratio of the ARG in the lactating sow). Figure S3: Proportion of Enterobacteriaceae resistant to sulfonamide (left) and tetracycline (right) in piglets at 14 days and at 4 weeks (i.e., weaning). Figure S4: Lactating sow effect on Enterobacteriaceae resistance to tetracycline in piglets at 4 weeks (weaning). Figure S5: Graphical representation of the impact of the S1 suspension on ARGs at 14 days and 4 weeks. Figure S6: Graphical representation of the impact of the S2 suspension on ARGs at 14 days and 4 weeks. Figure S7: Fitting the neutral model to the control, S1 and S2 piglets of sow 722. Figure S8: Breakdown of the frequency of the OTUs in S1, S2 and control piglets compared to the abundance of the OTUs in the lactating sows. Figure S9: Number of reads of the OTUs in the donor sows by their global contribution in the DAPC. Figure S10: Representative relationships found between OTUs and ARGs. Figure S11: Comparison of the distribution of the maximal information coefficient index in the dataset before and after randomization. Figure S12: Network of significant ARGs and OTU relationships at weaning.

Author Contributions: O.Z., C.S.A., V.D. and N.L.F. designed the study of the microbiota, N.L.F. performed the selection and sampling of the pigs, C.S.A. extracted the microbial DNA, chose the primers and analyzed the microbial sequences. L.C. uploaded the sequences. C.S.A. and O.Z. performed the statistical analyses. V.D. and N.A. screened the cultivable Enterobacteriacae for antibiotic resistance. V.D., A.B.-M., C.S.A., O.Z. and N.L.F. drafted the manuscript. All authors have read and agreed to the published version of the manuscript.

Funding: This research was funded by the Animal Health Program of the Carnot Institute, grant MICRORESET.

Acknowledgments: We thank the staff of the INRAE experimental unit of Saint Gilles (UEPR) for taking care of the pigs and the data and sample collection. This work was performed in collaboration with the GeT core facility, Toulouse, France (http://get.genotoul.fr), and was supported by France Génomique National infrastructure, funded as part of "Investissement d'avenir" program managed by the Agence Nationale pour la Recherche (contract ANR-10-INBS-09). We thank Helene Gilbert (INRAE) for her helpful discussions.

Conflicts of Interest: The authors declare no conflict of interest. The funders had no role in the design of the study, in the collection, analyses, or interpretation of data, in the writing of the manuscript, or in the decision to publish the results.

\section{References}

1. Xiao, L.; Estelle, J.; Kiilerich, P.; Ramayo-Caldas, Y.; Xia, Z.; Feng, Q.; Liang, S.; Pedersen, A.O.; Kjeldsen, N.J.; Liu, C.; et al. A reference gene catalogue of the pig gut microbiome. Nat. Microbiol. 2016. [CrossRef] [PubMed]

2. Gerzova, L.; Babak, V.; Sedlar, K.; Faldynova, M.; Videnska, P.; Cejkova, D.; Jensen, A.N.; Denis, M.; Kerouanton, A.; Ricci, A.; et al. Characterization of antibiotic resistance gene abundance and microbiota composition in feces of organic and conventional pigs from four EU countries. PLoS ONE 2015, 10, e0132892. [CrossRef] [PubMed]

3. Forslund, K.; Sunagawa, S.; Kultima, J.R.; Mende, D.R.; Arumugam, M.; Typas, A.; Bork, P. Country-specific antibiotic use practices impact the human gut resistome. Genome Res. 2013, 23, 1163-1169. [CrossRef] [PubMed]

4. Chen, Y.; Hammer, E.E.; Richards, V.P. Phylogenetic signature of lateral exchange of genes for antibiotic production and resistance among bacteria highlights a pattern of global transmission of pathogens between humans and livestock. Mol. Phylogenet. Evol. 2018, 125, 255-264. [CrossRef] [PubMed] 
5. Aarestrup, F. Get pigs off antibiotics. Nature 2012, 486, 465-466. [CrossRef]

6. Diana, A.; Manzanilla, E.G.; Calderón Díaz, J.A.; Leonard, F.C.; Boyle, L.A. Do weaner pigs need in-feed antibiotics to ensure good health and welfare? PLoS ONE 2017, 12, e0185622. [CrossRef] [PubMed]

7. Diana, A.; Boyle, L.A.; Leonard, F.C.; Carroll, C.; Sheehan, E.; Murphy, D.; Manzanilla, E.G. Removing prophylactic antibiotics from pig feed: How does it affect their performance and health? BMC Vet. Res. 2019, 15, 67. [CrossRef] [PubMed]

8. Cromwell, G.L. Why and how antibiotics are used in swine production. Anim. Biotechnol. 2002, 13, 7-27. [CrossRef]

9. Abreu, R.; Rodríguez-Álvarez, C.; Lecuona, M.; Castro, B.; González, J.C.; Aguirre-Jaime, A.; Arias, Á. Increased Antimicrobial Resistance of MRSA Strains Isolated from Pigs in Spain between 2009 and 2018. Vet. Sci. 2019, 6, 38. [CrossRef]

10. Zeitouni, S.; Kempf, I. Fitness cost of fluoroquinolone resistance in Campylobacter coli and Campylobacter jejuni. Microb. Drug Resist. 2012, 17, 171-179. [CrossRef]

11. Jernberg, C.; Lofmark, S.; Edlund, C.; Jansson, J.K. Long-term impacts of antibiotic exposure on the human intestinal microbiota. Microbiology 2010, 156, 3216-3223. [CrossRef] [PubMed]

12. Jernberg, C.; Lofmark, S.; Edlund, C.; Jansson, J.K. Long-term ecological impacts of antibiotic administration on the human intestinal microbiota. ISME J. 2007, 1, 56-66. [CrossRef] [PubMed]

13. Abecia, L.; Fondevila, M.; Balcells, J.; McEwanz, N.R. The effect of lactating rabbit does on the development of the caecal microbial community in the pups they nurture. J. Appl. Microbiol. 2007, 103, 557-564. [CrossRef] [PubMed]

14. Thompson, C.L.; Wang, B.; Holmes, A.J. The immediate environment during postnatal development has long-term impact on gut community structure in pigs. ISME J. 2008, 2, 739-748. [CrossRef]

15. Nurmi, E.; Rantala, M. New aspects of Salmonella infection in broiler production. Nature 1973, 241, $210-211$. [CrossRef]

16. Hofacre, C.L.; Johnson, A.C.; Kelly, B.J.; Froyman, R. Effect of a commercial competitive exclusion culture on reduction of colonization of an antibiotic-resistant pathogenic Escherichia coli in day-old broiler chickens. Avian Dis. 2002, 46, 198-202. [CrossRef]

17. Nuotio, L.; Schneitz, C.; Nilsson, O. Effect of competitive exclusion in reducing the occurrence of Escherichia coli producing extended-spectrum beta-lactamases in the ceca of broiler chicks. Poult. Sci. 2013, 92, 250-254. [CrossRef]

18. Ubeda, C.; Bucci, V.; Caballero, S.; Djukovic, A.; Toussaint, N.C.; Equinda, M.; Lipuma, L.; Ling, L.; Gobourne, A.; No, D.; et al. Intestinal microbiota containing Barnesiella species cures vancomycin-resistant Enterococcus faecium colonization. Infect. Immun. 2013, 81, 965-973. [CrossRef]

19. Chapman, B.C.; Moore, H.B.; Overbey, D.M.; Morton, A.P.; Harnke, B.; Gerich, M.E.; Vogel, J.D. Fecal microbiota transplant in patients with Clostridium difficile infection: A systematic review. J. Trauma Acute Care Surg. 2016, 81, 756-764. [CrossRef]

20. Staley, C.; Hamilton, M.J.; Vaughn, B.P.; Graiziger, C.T.; Newman, K.M.; Kabage, A.J.; Sadowsky, M.J.; Khoruts, A. Successful resolution of recurrent Clostridium difficile infection using freeze-dried, encapsulated fecal microbiota; pragmatic cohort study. Am. J. Gastroenterol. 2017. [CrossRef]

21. Anderson, R.C.; Stanker, L.H.; Young, C.R.; Buckley, S.A.; Genovese, K.J.; Harvey, R.B.; Deloach, J.R.; Keith, N.K.; Nisbet, D.J. Effect of competitive exclusion treatment on colonization of early-weaned pigs by Salmonella serovar Choleraesuis. J. Swine Health Prod. 1999, 7, 155-160.

22. Genovese, K.J.; Anderson, R.C.; Harvey, R.B.; Callaway, T.R.; Poole, T.L.; Edrington, T.S.; Fedorka-Cray, P.J.; Nisbet, D.J. Competitive exclusion of Salmonella from the gut of neonatal and weaned pigs. J. Food Prot. 2003, 66, 1353-1359. [CrossRef] [PubMed]

23. Kim, L.M.; Gray, J.T.; Bailey, J.S.; Jones, R.D.; Fedorka-Cray, P.J. Effect of porcine-derived mucosal competitive exclusion culture on antimicrobial resistance in Escherichia coli from growing piglets. Foodborne Pathog. Dis. 2005, 2, 317-329. [CrossRef] [PubMed]

24. Luyckx, K.; Millet, S.; Van Weyenberg, S.; Herman, L.; Heyndrickx, M.; Dewulf, J.; De Reu, K. Comparison of competitive exclusion with classical cleaning and disinfection on bacterial load in pig nursery units. BMC Vet. Res. 2016, 12. [CrossRef] 
25. Verschuren, L.M.G.; Calus, M.P.L.; Jansman, A.J.M.; Bergsma, R.; Knol, E.F.; Gilbert, H.; Zemb, O. Fecal microbial composition associated with variation in feed efficiency in pigs depends on diet and sex. J. Anim. Sci. 2018, 96, 1405-1418. [CrossRef]

26. Eucast. Available online: http://www.eucast.org/ (accessed on 14 May 2015).

27. Le Floc'h, N.; Knudsen, C.; Gidenne, T.; Montagne, L.; Merlot, E.; Zemb, O. Impact of feed restriction on health, digestion and faecal microbiota of growing pigs housed in good or poor hygiene conditions. Animal 2014, 8, 1632-1642. [CrossRef]

28. Magoc, T.; Salzberg, S.L. FLASH: Fast length adjustment of short reads to improve genome assemblies. Bioinformatics 2011, 27, 2957-2963. [CrossRef]

29. NCBI Sequence Read Archive. Available online: https://trace.ncbi.nlm.nih.gov/Traces/sra/sra.cgi (accessed on 7 October 2018).

30. Edgar, R.C. UPARSE: Highly accurate OTU sequences from microbial amplicon reads. Nat. Methods 2013, 10, 996-998. [CrossRef]

31. McMurdie, P.J.; Holmes, S. Phyloseq: An R package for reproducible interactive analysis and graphics of microbiome census data. PLoS ONE 2013, 8, e61217. [CrossRef]

32. Jombart, T.; Devillard, S.; Balloux, F. Discriminant analysis of principal components: A new method for the analysis of genetically structured populations. BMC Genet. 2010, 11, 94. [CrossRef]

33. Burns, A.R.; Stephens, W.Z.; Stagaman, K.; Wong, S.; Rawls, J.F.; Guillemin, K.; Bohannan, B.J. Contribution of neutral processes to the assembly of gut microbial communities in the zebrafish over host development. ISME J. 2016, 10, 655-664. [CrossRef] [PubMed]

34. Reshef, D.N.; Reshef, Y.A.; Finucane, H.K.; Grossman, S.R.; McVean, G.; Turnbaugh, P.J.; Lander, E.S.; Mitzenmacher, M.; Sabeti, P.C. Detecting Novel Associations in Large Data Sets. Science 2011, 334, 1518-1524. [CrossRef] [PubMed]

35. Leser, T.D.; Amenuvor, J.Z.; Jensen, T.K.; Lindecrona, R.H.; Boye, M.; Moller, K. Culture-independent analysis of gut bacteria: The pig gastrointestinal tract microbiota revisited. Appl. Environ. Microbiol. 2002, 68, 673-690. [CrossRef] [PubMed]

36. Pang, X.; Hua, X.; Yang, Q.; Ding, D.; Che, C.; Cui, L.; Jia, W.; Bucheli, P.; Zhao, L. Inter-species transplantation of gut microbiota from human to pigs. ISME J. 2007, 1, 156-162. [CrossRef]

37. Jansman, A.J.M.; Zhang, J.; Koopmans, S.J.; Dekker, R.A.; Smidt, H. Effects of a simple or a complex starter microbiota on intestinal microbiota composition in caesarean derived piglets. J. Anim. Sci. 2012, 90, 433-435. [CrossRef]

38. Gleed, P.; Sansom, B. Ingestion of iron in sows feces by piglets in farrowing crates with slotted floors. Br. J. Nutr. 1982, 47, 113-117. [CrossRef]

39. Schmidt, B.; Mulder, I.E.; Musk, C.C.; Aminov, R.I.; Lewis, M.; Stokes, C.R.; Bailey, M.; Prosser, J.I.; Gill, B.P.; Pluske, J.R.; et al. Establishment of normal gut microbiota is compromised under excessive hygiene conditions. PLoS ONE 2011, 6, e28284. [CrossRef]

40. Hu, J.; Ma, L.; Nie, Y.; Chen, J.; Zheng, W.; Wang, X.; Xie, C.; Zheng, Z.; Wang, Z.; Yang, T.; et al. A Microbiota-Derived Bacteriocin Targets the Host to Confer Diarrhea Resistance in Early-Weaned Piglets. Cell Host Microbe 2018, 24, 817-832. [CrossRef]

41. Volf, J.; Polansky, O.; Varmuzova, K.; Gerzova, L.; Sekelova, Z.; Faldynova, M.; Babak, V.; Medvecky, M.; Smith, A.L.; Kaspers, B.; et al. Transient and prolonged response of chicken cecum mucosa to colonization with different gut microbiota. PLoS ONE 2016, 11, e0163932. [CrossRef]

42. Achard, C.S.; Dupouy, V.; Siviglia, S.; Arpaillange, N.; Cauquil, L.; Bousquet-Mélou, A.; Zemb, O. Variability of the Ability of Complex Microbial Communities to Exclude Microbes Carrying Antibiotic Resistance Genes in Rabbits. Front. Microbiol. 2019, 10, 1503. [CrossRef]

43. Kiros, T.G.; Derakhshani, H.; Pinloche, E.; D’Inca, R.; Marshall, J.; Auclair, E.; Khafipour, E.; Van Kessel, A. Effect of live yeast Saccharomyces cerevisiae (Actisaf Sc 47) supplementation on the performance and hindgut microbiota composition of weanling pigs. Sci. Rep. 2018, 8, 5315. [CrossRef] [PubMed]

44. Mu, C.; Bian, G.; Su, Y.; Zhu, W. Differential Effects of Breed and Nursing on Early-Life Colonic Microbiota and Immune Status as Revealed in a Cross-Fostering Piglet Model. Appl. Environ. Microbiol. 2019, 85. [CrossRef] [PubMed] 
45. Dominguez-Bello, M.G.; De Jesus-Laboy, K.M.; Shen, N.; Cox, L.M.; Amir, A.; Gonzalez, A.; Bokulich, N.A.; Song, S.J.; Hoashi, M.; Rivera-Vinas, J.I.; et al. Partial restoration of the microbiota of cesarean-born infants via vaginal microbial transfer. Nat. Med. 2016, 22, 250-253. [CrossRef] [PubMed]

46. Schokker, D.; Zhang, J.; Vastenhouw, S.A.; Heilig, H.G.H.J.; Smidt, H.; Rebel, J.M.J.; Smits, M.A. Long-Lasting Effects of Early-Life Antibiotic Treatment and Routine Animal Handling on Gut Microbiota Composition and Immune System in Pigs. PLoS ONE 2015, 10, e0116523. [CrossRef] [PubMed]

47. Stanton, T.B.; Humphrey, S.B. Persistence of Antibiotic Resistance: Evaluation of a Probiotic Approach Using Antibiotic-Sensitive Megasphaera elsdenii Strains to Prevent Colonization of Swine by Antibiotic-Resistant Strains. Appl. Environ. Microbiol. 2011, 77, 7158-7166. [CrossRef]

48. Holman, D.B.; Chénier, M.R. Impact of subtherapeutic administration of tylosin and chlortetracycline on antimicrobial resistance in farrow-to-finish swine. FEMS Microbiol. Ecol. 2013, 85, 1-13. [CrossRef]

49. Werner, G.; Hildebrandt, B.; Witte, W. Linkage of erm(B) and aadE-sat4-aphA-3 in multiple-resistant Enterococcus faecium isolates of different ecological origins. Microb. Drug Resist. 2003, 9 (Suppl. 1), S9-S16. [CrossRef]

50. Kalmokoff, M.; Waddington, L.M.; Thomas, M.; Liang, K.L.; Ma, C.; Topp, E.; Dandurand, U.D.; Letellier, A.; Matias, F.; Brooks, S.P. Continuous feeding of antimicrobial growth promoters to commercial swine during the growing/finishing phase does not modify faecal community erythromycin resistance or community structure. J. Appl. Microbiol. 2011, 110, 1414-1425. [CrossRef]

51. Birkegård, A.C.; Halasa, T.; Græsbøll, K.; Clasen, J.; Folkesson, A.; Toft, N. Association between selected antimicrobial resistance genes and antimicrobial exposure in Danish pig farms. Sci. Rep. 2017, 7, 9683. [CrossRef]

52. Looft, T.; Johnson, T.A.; Allen, H.K.; Bayles, D.O.; Alt, D.P.; Stedtfeld, R.D.; Sul, W.J.; Stedtfeld, T.M.; Chai, B.; Cole, J.R.; et al. In-feed antibiotic effects on the swine intestinal microbiome. PNAS 2012, 109, 1691-1696. [CrossRef]

53. Swords, W.E.; Wu, C.C.; Champlin, F.R.; Buddington, R.K. Postnatal changes in selected bacterial groups of the pig colonic microflora. Biol. Neonate 1993, 63, 191-200. [CrossRef] [PubMed]

54. Johnson, T.A.; Stedtfeld, R.D.; Wang, Q.; Cole, J.R.; Hashsham, S.A.; Looft, T.; Zhu, Y.G.; Tiedje, J.M. Clusters of antibiotic resistance genes enriched together stay together in swine agriculture. MBio 2016, 7, e02214-e02215. [CrossRef] [PubMed]

55. Hegstad, K.; Mikalsen, T.; Coque, T.M.; Werner, G.; Sundsfjord, A. Mobile genetic elements and their contribution to the emergence of antimicrobial resistant Enterococcus faecalis and Enterococcus faecium. Clin. Microbiol. Infect. 2010, 16, 541-554. [CrossRef] [PubMed]

56. Roberts, M.C. Update on macrolide-lincosamide-streptogramin, ketolide, and oxazolidinone resistance genes. FEMS Microbiol. Lett. 2008, 282, 147-159. [CrossRef]

57. Bayley, D.P.; Rocha, E.R.; Smith, C.J. Analysis of cepA and other Bacteroides fragilis genes reveals a unique promoter structure. FEMS Microbiol. Lett. 2000, 193, 149-154. [CrossRef]

58. Raftis, E.J.; Forde, B.M.; Claesson, M.J.; O’Toole, P.W. Unusual genome complexity in Lactobacillus salivarius JCM1046. BMC Genom. 2014, 15, 771. [CrossRef]

(C) 2020 by the authors. Licensee MDPI, Basel, Switzerland. This article is an open access article distributed under the terms and conditions of the Creative Commons Attribution (CC BY) license (http://creativecommons.org/licenses/by/4.0/). 